\title{
Environmental impact of soluble oils
}

\author{
S. Riley ${ }^{1}$, C. McQuade ${ }^{2}$, S. Adeloju ${ }^{3} \&$ R. Hyne ${ }^{4}$

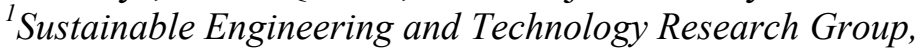 \\ University of Western Sydney, Australia \\ ${ }^{2}$ Business Support, Zinifex Port Pirie Smelter, Australia \\ ${ }^{3}$ School of Applied Science and Engineering, \\ Monash University, Gippsland Campus, Australia \\ ${ }^{4}$ Department of Environment and Climate Change, Sydney, Australia
}

\begin{abstract}
The objective of the study was to determine the level of soluble oil at which the environmental risk of its discharge is unacceptable. Specifically, the potential toxicity to aquatic species of mine water discharge containing soluble hydraulic oil emulsions was determined. The study was undertaken at a mine site in the Western Coal Fields of the Blue Mountains, Australia, and involved risk analysis and toxicity and chemical testing of water discharged at the pit head. Some initial problems were encountered in toxicity testing when the background stream waters draining a relatively undisturbed catchment proved to be toxic to the test organisms. This toxicity was determined to be related to fine-grained sediment in the water. The study supported the soluble oil manufacturer's claims that there is a 90 to $95 \%$ decrease in soluble oil concentrations as a result of biodegradation over a 28 day period. In normal mine operations soluble oil appears to biodegrade before it reaches the surface. Normal soluble oil usage in mine operations does not appear to be a risk to the environment unless there is a substantial spill that is then pumped to the surface and discharged directly into receptor streams. Such spills are rare, but have been known to occur
\end{abstract}

Keywords: soluble oil, mine water toxicity, environmental impact of mines.

\section{Introduction}

Soluble hydraulic oil is an integral component of all longwall operations, typically used as a five percent solution with water. The daily quantity used in 
the longwall mining of coal can vary from as little as $50 \mathrm{~L}$ to greater than $1200 \mathrm{~L}$, depending upon the age, technology and maintenance of the equipment. Soluble oil is recognised as an agent that removes the natural oil from the skin, which in turn may lead to skin irritation and potentially longer term skin damage. Soluble oil, when discharged from a mining operation, may also impact on aquatic ecosystems. The degree of potential aquatic ecosystem impact is undefined for Australian environments.

Soluble oils, while they biodegrade to water and carbon dioxide, are difficult and expensive to remove from water as an "end of pipe" control. Furthermore, the rate of biodegradation is unknown for many of the waters encountered in mine pits. Where surfactants are a component of the oils there is considerable potential for a white froth to develop in areas of water aeration. Such effects can be visually spectacular, but highly undesirable for the mining industry.

There is currently little known, within Australia and Internationally, about the environmental effects of soluble oils in the mining industry and how to manage the risks associated with these products (Sprince et al. [1]; Barron, et al. [2]), even thought there are numerous studies of the toxicity of soluble components of oils. This project was formulated in the context of the lack of information and within the wider context of the need to be proactive in reducing uncertainties about environmental impacts of mining operations.

The project was approved and supported by the Australian Coal Association Research Program ACARP, project C7034. The aims of the project were to establish whether mine water discharges, which may have been contaminated with soluble hydraulic oil emulsions, are toxic to aquatic species and could impact on the environment of receptor streams and the level of soluble oil at which environmental risk of discharge is unacceptable - a proof of concept study.

\section{Soluble oils}

Soluble oils, such as Solsenic, are used in the mines to form highly stable oil-inwater emulsions that are capable of providing efficient lubrication properties, as well as cleanliness and stability. Material Safety Data Sheets for soluble oils typically include rates of biodegradability and occasionally toxicity of their products. The issues with this information are that biodegradability tests are often assessed using sewage sludge as the biological innoculant. These tests, while representing an Australian and International standard, bear no or little resemblance to the rate of biodegradability in underground mining operations or the natural stream systems of Australia. A rapid biodegrading oil does not directly infer an environmentally friendly product. The biodegradation requires oxygen, with the possible depletion of oxygen in receiving waters and consequent death of sensitive species. There may be other potentially toxic agents arising from the biodegradation. The selection and application of soluble oils for the specific mining environment is a consideration in overall protection of the environment. 


\section{Method}

The experimental method involved sampling the discharge water from a representative mine site in the Western Sydney Basin, dosing this water with soluble oil and determining the toxicity of different doses and the biodegradation rate of the oils. Chemical tests were undertaken to characterise the geochemical nature of the water and its organic content. The essential fabric of the experimental method is outlined in Figure 1.

The primary outcome of this project, undertaken as a feasibility study, was to determine the level of soluble oil concentration at which the environmental risk of discharge is unacceptable. Environmental risk is defined by the toxicity of the water-soluble oil mix and the key physio-chemical parameters.

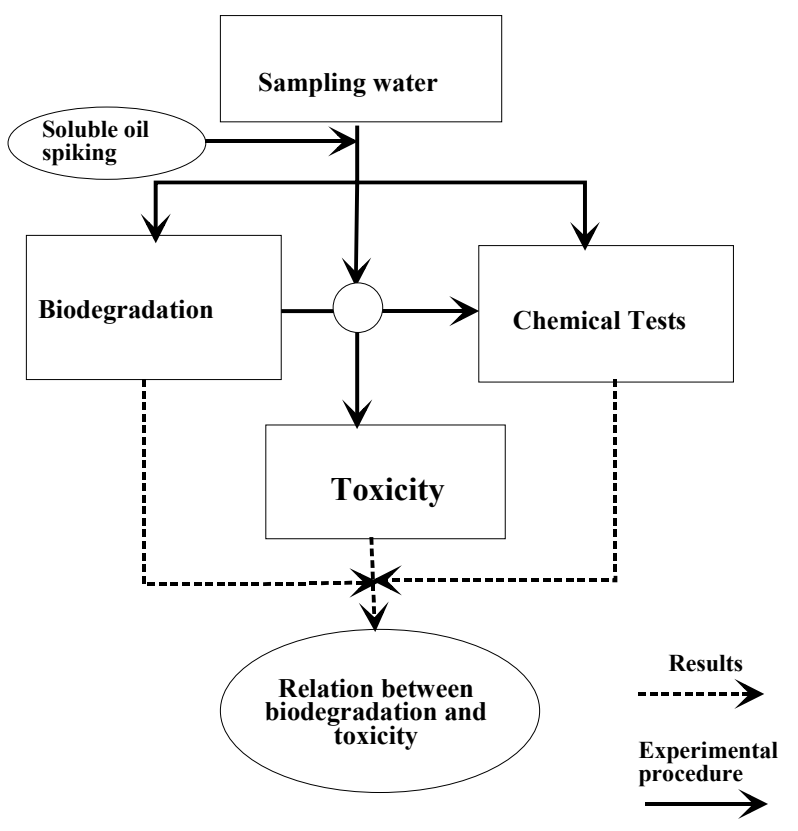

Figure 1: Schematic diagram showing the program of the proof of concept study.

Intitial work centred around end-of-pipe discharges to the environment. These discharges were assayed for chemical make-up, biochemical activity and toxicity toward aquatic species. A variety of tests and determinations were conducted. These tests were repeated, where appropriate, on discharge water spiked with the soluble oil used by the chosen mine site. The tests were undertaken at relevant stages of biodegradation.

The soluble oil brand name analysed in this study was Solcenic. The manufacturer of the product is Century Oils. The material supplied is official known as Soluble HL concentrate, product code U291. 
The extent of biodegradability of soluble oils in the water and the presence of other inorganic and organic substances was monitored by analysing for the following substances:

- $\quad$ soluble hydrocarbons (C6-C9, C10-C14, C15-C28, C29-C36) fractions;

- $\quad$ oil and grease;

- $\quad$ polynuclear aromatic hydrocarbons;

- $\quad$ heavy metals;

- $\quad \mathrm{pH}$, conductivity and dissolved oxygen.

Previous work on water quality suggests that acid mine drainage and water quality of discharges from Western Blue Mountains (Western District) mines are unlikely to be an environmental problem, except for the metal zinc concentration (Toyer and Main [3]). Zinc is usually present in significantly high concentrations in some mine waters.

The concentration of soluble oil in pit head discharge from the Colliery was estimated from mine records of water discharge from underground and soluble oil usage figures (Fig 2).

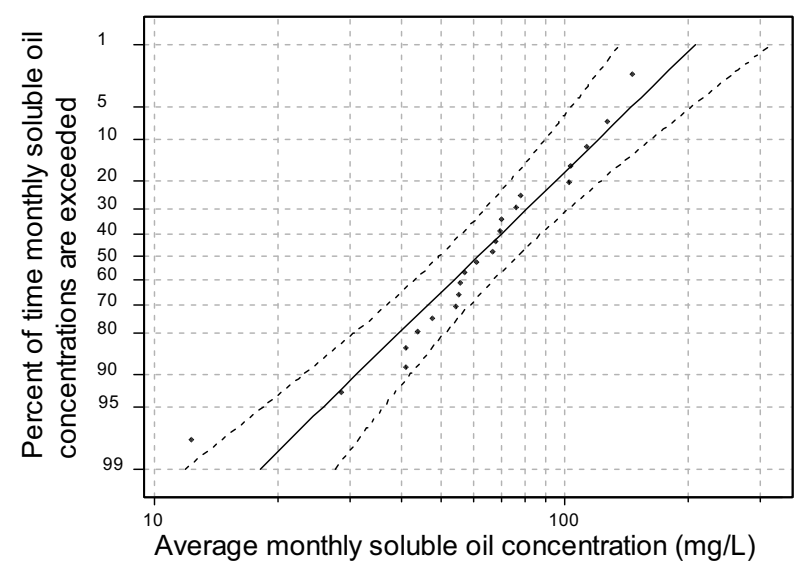

Figure 2: $\quad$ Probability analysis of maximum soluble oil concentration at the pit head ignoring degradation of the soluble oil.

The toxicity test (Fig 3 ) involved exposing Ceriodaphnia dubia neonates $(<24$ $\mathrm{h}$ old) to a series of concentrations of mine water, for $48 \mathrm{hrs}$ under static conditions, to determine a $48 \mathrm{~h}$ LC50 value. Further details of experimental method are provided in Lim et al. [4]. Dechlorinated Sydney tap water was used as a control water. The first set of tests were conducted on water being discharged from the mine, with a set of 3 tests to assess temporal trends. If no effects were detected then single tests were conducted on water spiked with oil to identify worse-case situation and 10 times worse case situation. For the latter a temporal set of tests (1 to 30 days) were undertaken. If there was no effect then no further testing was required. 


\section{Phase 1}
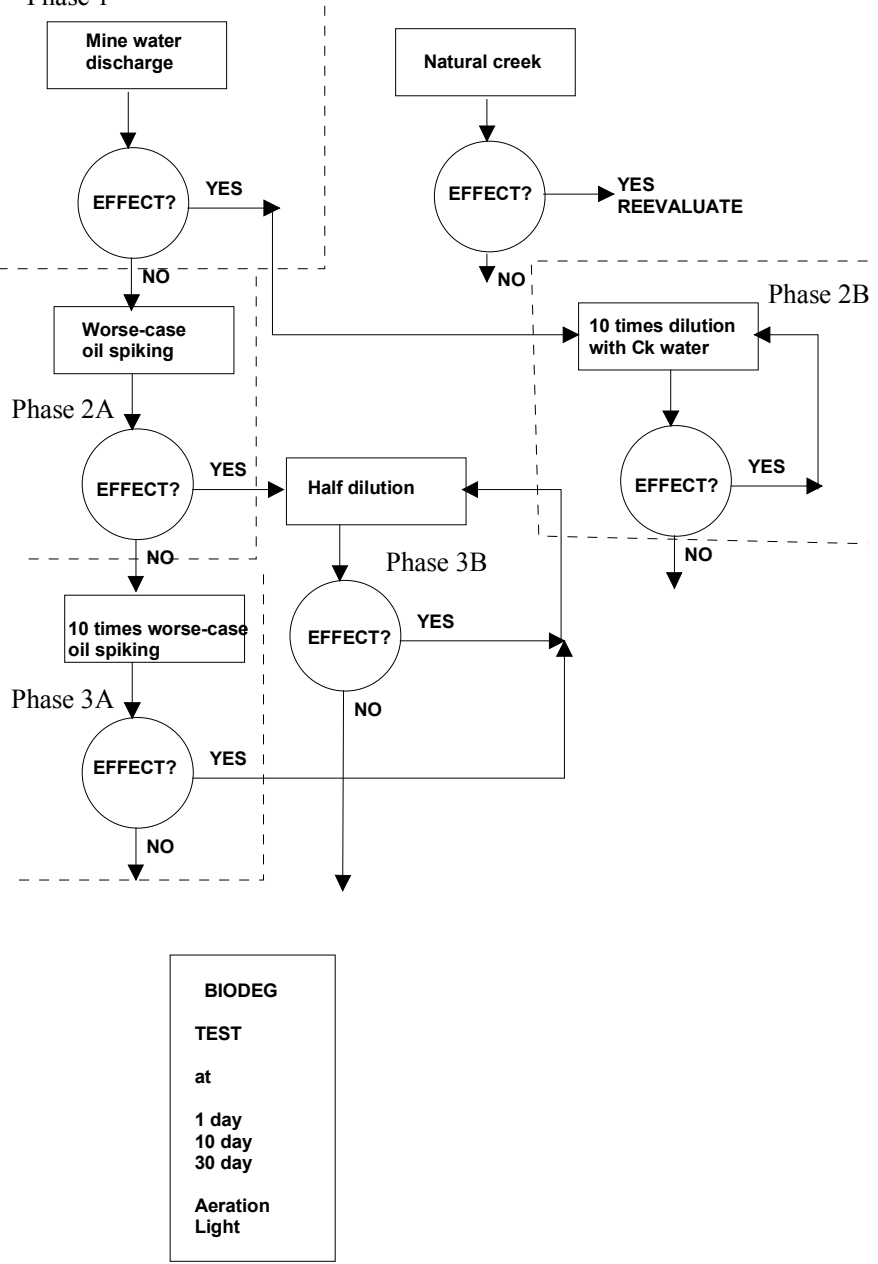

Figure 3: $\quad$ Toxicity testing strategy for the project.

Throughout the sampling period the pit head discharge water exhibited a neutral $\mathrm{pH}$ and low levels of iron, manganese, zinc and potassium were found. The presence of organic species in the pit head discharge water was also identified, but the concentrations were found to be quite variable.

\section{Results}

In the first phase of the project to determine the toxicity of mine water to the cladoceran, Ceriodaphnia dubia the water was found to be toxic. However, it was not known whether soluble oil was contributing to the toxicity of the water. To assess whether soluble oil was contributing to its toxicity a Toxicity Identification Evaluation (TIE) study was conducted by exposing freshwater 
cladoceran Ceriodaphnia dubia to the mine water. The mine water sample was initially tested to determine if the sample was acutely toxic using Whole Effluent Toxicity (WET) test procedures. If toxicity was observed, a TIE was then undertaken to identify the cause(s) of the toxicity of the mine water. In addition the degradation test was repeated with another sample of mine water to confirm the findings of the test conducted in the first phase of the project. TIE procedures have been summarised in a series of documents by the USEPA [5-8]. The TIE approach is divided into three phases. Phase I utilises effluent manipulations to characterise the class of contaminant (e.g. non-polar organics, metals, ammonia) causing the observed toxicity. Phase II of the TIE process further identifies the toxicant within the class of contaminants identified in Phase I, and Phase III includes confirmation procedures for suspect toxicants. These procedures are based on an iterative approach, in which TIE manipulations are chosen on the basis of results from previous tests and any other available information.

The mine water sample, which was used for the TIE, was tested only for acute toxicity. This sample exhibited no toxicity at 24 hours exposure, but exhibited partial mortalities in the undiluted $(100 \%)$ and $50 \%$ sample dilution treatments. The 48 hour LC50 and 95\% confidence interval of this sample was estimated to be $73.5 \%(59.0-91.6 \%)$ of the mine water. This was much less toxic compared with that of the mine water samples used in first phase of the project which had LC50 values between 25 and $28 \%$ of the mine water

As the mine water was toxic to the cladoceran a TIE was initiated. This was to determine if soluble oil was the cause of the acute toxicity exhibited by this sample. The endpoint was mortality.

The mine water sample was passed through a C18 Solid Phase Extraction (SPE) column and the filtrate tested for toxicity. In addition, the SPE column was eluted with methanol and the eluant spiked into dilution water at a concentration representing 1-times and 2-times the original concentration in the sample (referred to as $1 \mathrm{X}$ and $2 \mathrm{X}$ add-backs). The SPE filtrate was not toxic at 48 hours (Table 13.4), and it can be concluded that the toxicant had adhered to the SPE column matrix. However, the methanol eluate was also not toxic at either $1 \mathrm{X}$ or $2 \mathrm{X}$ add-back concentrations, which suggest that the toxicant was not mobilised by a low polar solvent. This would indicate that the toxicant was not a non-polar organic compound. It was also apparent that the toxicity of the sample was dissipating, as the $50 \%$ mine water treatment was no longer toxic, and that the undiluted mine water caused $60 \%$ mortality compared with $70 \%$ in the original WET test.

In a second Phase I TIE manipulation, EDTA was added to the mine water sample at 75, 150 and $200 \mathrm{mg} / \mathrm{L}$. This treatment eliminated the toxicity of the sample at 48 hours, which suggests that a divalent metal(s) was responsible for the observed toxicity (Table 1).

Some divalent metals such as copper and zinc can adhere to the matrix of an SPE column, but can not be eluted by low polarity solvents, such as methanol. Thus, the results obtained by the SPE extraction/elution procedure and the EDTA addition manipulations suggest that a divalent metal was responsible for the observed toxicity. 
Table 1: $\quad$ Phase I TIE manipulations with mine water: EDTA addition.

\begin{tabular}{|l|c|c|}
\hline \multicolumn{1}{|c|}{ Treatment } & \% mortality at 24 hrs & \% mortality at 48 hrs \\
\hline \multicolumn{1}{|c|}{ Control } & 0 & 0 \\
\hline EDTA control $(75 \mathrm{mg} / \mathrm{L})$ & 10 & 10 \\
\hline EDTA control $(150 \mathrm{mg} / \mathrm{L})$ & 0 & 20 \\
\hline EDTA control $(200 \mathrm{mg} / \mathrm{L})$ & 0 & 0 \\
\hline $100 \%$ mine water & 60 & 0 \\
\hline $50 \%$ mine water & 0 & 10 \\
\hline $\begin{array}{l}100 \% \text { mine water }+75 \mathrm{mg} \\
\text { EDTA/L }\end{array}$ & 0 & 10 \\
\hline $\begin{array}{l}100 \% \text { mine water }+150 \mathrm{mg} \\
\text { EDTA/L }\end{array}$ & 0 & 30 \\
\hline $\begin{array}{l}100 \% \text { mine water }+200 \mathrm{mg} \\
\text { EDTA/L }\end{array}$ & 0 & \\
\hline
\end{tabular}

A sub sample of the mine water was subject to an ICP/MS measurement to identify the presence of heavy metals. Total and filtered zinc concentrations were detected at 0.14 and $0.068 \mathrm{mg} / \mathrm{L}$ respectively. The total zinc concentration found was nearly twice as high as the $48 \mathrm{hr}$ LC50 of $\mathrm{Zn}$ to $C$. dubia $(0.073 \mathrm{mg} / \mathrm{L})$ determined in the first phase of the project. In addition, the $48 \mathrm{hr}$ LC50 of zinc (total) to C. dubia in EPA synethetic water has been shown to vary between 0.07 and $0.20 \mathrm{mg} / \mathrm{L}$ (Hyne et al. [9]). This suggests that zinc was a likely suspect for the observed toxicity.

To eliminate soluble oil as a suspect toxicant, similar TIE treatments were performed with soluble oil as were undertaken with the mine water sample. A 48 hour acute toxicity test was performed on the soluble oil, and $100 \%$ mortalities were observed at $0.001 \%(\mathrm{v} / \mathrm{v})$ soluble oil in laboratory water (Table 2). The 48 hr LC50 and 95\% confidence limits were estimated to be $0.0007 \%(0.0006$ $0.0008 \%$ ) soluble oil. This is similar to the values obtained in the first phase of the project $(0.0005-0.00076 \%)$.

Table 2: $\quad$ Mortality of cladocerans exposed to soluble oil.

\begin{tabular}{|c|c|c|}
\hline Treatment (\% soluble oil) & $\%$ mortality at $24 \mathrm{hrs}$ & $\%$ mortality at $48 \mathrm{hrs}$ \\
\hline 0 (control) & 0 & 5 \\
\hline 0.0000625 & 0 & 0 \\
\hline 0.000125 & 0 & 0 \\
\hline 0.00025 & 0 & 0 \\
\hline 0.0005 & 0 & 10 \\
\hline 0.001 & 65 & 90 \\
\hline
\end{tabular}

Passing a $0.001 \%$ solution of soluble oil through a C18 SPE column failed to remove toxicity in the SPE filtrate. The addition of EDTA failed to reduce the toxicity of $0.001 \%$ soluble oil. However, some of the soluble oil was bound to the SPE matrix, as $1 \mathrm{X}$ and $2 \mathrm{X}$ add-back concentrations also demonstrated 
toxicity, and SPE filtrate exhibited reduced toxicity at 24 hrs. This preliminary Phase I fingerprint for soluble oil does not match that of the toxicant present in the mine water sample, and suggests that soluble oil was not responsible for the toxicity exhibited in the mine water sample.

\section{Discussion and conclusion}

This study indicated that the levels of soluble oils used in the mine did not contribute to the toxicity of the mine water as demonstrated by the TIE approach. A preliminary Phase I fingerprint for soluble oil did not match that of the toxicant present in the mine water sample further suggesting that soluble oil was not responsible for the toxicity exhibited in the mine water sample. The results however, suggest that zinc is the cause of toxicity in the mine water.

Passing the water through a C18 Solid Phase Extraction (SPE) column eliminated toxicity from the filtrate. However, eluting the SPE column failed to remove the toxicant. Divalent metals such as copper and zinc are known to adhere to SPE matices, but are not readily eluted by solvents such as methanol.

Adding EDTA to the effluent eliminated the toxicity of the mine water, suggesting that the toxicant was a divalent metal. Analysis of the mine water detected total and dissolved zinc concentration in the sample at 0.14 and 0.068 $\mathrm{mg} / \mathrm{L}$ respectively. This concentration of zinc has previously been demonstrated to cause acute toxicity to Ceriodaphnia dubia. This suggests that zinc was a likely suspect chemical. However the sample had lost its toxicity by this time, and a confirmation study could not be undertaken.

To eliminate soluble oil as a suspect toxicant, similar TIE treatments were conducted on a $0.001 \%$ (approximately $10 \mathrm{mg} / \mathrm{L}$ ) soluble oil solution as was conducted on the mine water. Both SPE extraction and EDTA addition failed to eliminate acute toxicity to $C$. dubia, suggesting that soluble oil was not responsible for the toxicity observed in the mine water sample.

The biodegradation of the soluble oil appears to be very efficient. All the evidence suggests that the manufacturer's claim of $90 \%$ or more biodegradation of the soluble oil over a period of 28 days is supported. Hence, the largest problems facing mines appear to be substantial soluble oil discharges into waters which are pumped to the surface in short periods of time after spills occur.

\section{Acknowledgements}

We would like to thank the steering committee, Dr Ross Hyne, Mr Dick Wilkinson, Mr Paul Fredrickson, Dr Patrick McBridge, Mr Phil Leeming and Mr Peter Doyle.

\section{References}

[1] Sprince, N.L., Palmer, J.A., Popendorf, W., Thorne, P.S., m Selim, M.I., Zwerling, C., Miller, E.R. 1996. Dermatitis among automobile production 
machine operators exposed to metal-working fluids. American $J$ Industrial Medicine, 30(4),421-429.

[2] Barron, M.G., Podrabsky, T., Ogle,S., Ricker,R.W., 1999. Are aromatic hydrocarbons the primary determinant of petroleum toxicity to aquatic organisms. Aquatic Toxicology, 46(3-4), 253-268.

[3] Toyer, G.S and Main, S. 1981. Environmental implications of future underground cola mining developments on the Newnes Plateau, NSW, with particular emphasis on regional water quality aspects. Geological Survey of NSW Department of Mineral Resources Geological Survey Report No. GS1981/242 (revised 1983).

[4] Lim, R.P., Hyne, R.V., Doyle, C., Adeloju, S.B., Riley, S., and Cohen, D. 1999. Toxicity of soluble oil in mine water from Angus Place Colliery, NSW. Unpublished report 21pp.

[5] U.S. EPA (U.S. Environment Protection Agency). 1991. Methods for aquatic toxicity identification evaluations. Phase I toxicity characterisation procedures, 2nd ed. EPA-600/6-91/0303. Office of Research and Development, Duluth, MN.

[6] U.S. EPA. 1993a. Methods for measuring the acute toxicity of effluents and receiving waters to freshwater and marine organisms. 4th edition. C.I. Weber (ed). Environmental Monitoring and Support Laboratory. U.S. Environment Protection Agency, Cincinnati, OH. EPA/600/4-90/027F. 293 pp.

[7] U.S. EPA. 1993b. Methods for aquatic toxicity identification evaluations. Phase II toxicity identification procedures for samples exhibiting acute and chronic toxicity. EPA-600/R-92/080. Office of Research and Development, Duluth, MN.

[8] U.S. EPA. 1993c. Methods for aquatic toxicity identification evaluations. Phase III toxicity confirmation procedures for samples exhibiting acute and chronic toxicity. EPA-600/R-92/081. Office of Research and Development, Duluth, MN.

[9] Hyne, R.V., Pablo, F., Julli, M., Markich, S.J. 2005. Effect of solution parameters on the bioavailability and acute toxicity of copper and zinc to the cladoceran Ceriodaphnia cf dubia. Environ. Toxicol. Chem. 24: 1667-1675. 